\title{
Erratum to: Changing Lone Parents, Changing Life Courses
}

\author{
Laura Bernardi, Dimitri Mortelmans, and Ornella Larenza
}

\section{Erratum to:}

\section{Chapter 1 in: L. Bernardi, D. Mortelmans (eds.) Lone Parenthood in the Life Course, Life Course Research and Social Policies 8, https://doi.org/10.1007/978-3-319-63295-7_1}

The original version of this book was published without Ornella Larenza listed among the chapter authors in Chapter 1.

It has been corrected and updated as:

Laura Bernardi, Dimitri Mortelmans, and Ornella Larenza 